\title{
Light and electron microscopical studies of focal glomerular sclerosis
}

\author{
A. H. NAGI, F. ALEXANDER, AND R. LANNIGAN \\ From the Department of Pathology, Queen's University of Belfast
}

SYNOPSIS Renal biopsy material from seven cases of the nephrotic syndrome due to focal glomerular sclerosis has been studied by light, electron, and immunofluorescent microscopy. The nature of glomerular basement membrane changes and the scar tissue was also studied. It was found that the glomerular basement membrane and mesangial matrix formed the major components of scar tissue. On the basis of a short history in some of our cases, a poor response to steroid therapy in the early stages, and the distinct morphological changes, it is suggested that focal glomerular sclerosis has an independent origin and is not a stage of minimal change lesion.

Focal glomerular sclerosis is characterized by the presence of glomerular sclerosis which may be focal or segmental in distribution. Rich (1957) for the first time described 20 cases (all children) of what he called 'intercapillary or membranous glomerulosclerosis'. He thought that with the passage of time in some lesions of minimal change the basement membrane of the glomerular tufts tends to become increasingly thickened with the formation of capsular adhesions in some of them. These changes were characteristically seen in the juxtamedullary region. Williams (1965) reported a similar series in children and confirmed the findings of Rich. Following these two reports various other investigators (McGovern, 1967; White, 1970; White, Glasgow, and Mills, 1970; Churg, Habib, and White, 1970) have also recognized this change in some cases of the nephrotic syndrome, and thought it to be a different type of lesion from that of minimal change. White et al (1970) and Churg et al (1970) found that most of the cases of focal glomerular sclerosis are resistant to steroid therapy. The ultrastructural changes in various stages of focal glomerular sclerosis have not been previously described.

This paper describes the results of histological, electronmicroscopical, and immunofluorescent studies in seven cases of focal glomerular sclerosis. The effect of steroid therapy on these cases is also discussed.

${ }^{2}$ Correspondence should be addressed to Dr A. H. Nagi, Department of Pathology, Lahore General Hospital, Lahore, W. Pakistan.

Received for publication 9 June 1971.

\section{Materials and Methods}

The renal tissues from all the patients were obtained by open renal biopsies. Before operation each patient was investigated for renal function (including an intravenous pyelogram). Each biopsy was divided into three portions for light, electron, and immunofluorescent microscopy. The specimens were treated as follows.

\section{LIGHT MICROSCOPY}

The tissue was fixed in $10 \%$ formol-saline and embedded in paraffin. Each block was cut into sections 3-4 $\mu$ thick which were stained with haematoxylin and eosin, periodic acid-Schiff reaction, methenamine-silver, and Lendrum's acid picroMallory stains.

\section{ELECTRON MICROSCOPY}

Small blocks (three to five in number) of renal tissue were fixed in $5 \%$ glutaraldehyde, washed in sucrose cacodylate buffer, and postfixed in Caulfield's osmium tetroxide, dehydrated, and embedded in Epon. The glomeruli were selected from $1 \mu$ thick sections stained with toluidine-blue. Ultra-thin sections of three to five glomeruli from each case were cut and stained with uranyl-acetate and lead citrate. The sections were examined on uncoated grids on an EM-GB6 electron microscope.

\section{IMMUNOFLUORESCENT MICROSCOPY}

A third portion from each renal biopsy was wrapped 
in parafilm and kept at $-20^{\circ} \mathrm{C}$. Sections, $4 \mu$ thick, were cut using a cryostat and fixed in $4 \%$ buffered formol-saline, $p \mathbf{H} \mathbf{7 \cdot 2}$, for two to three minutes. These sections were stained using monospecific antihuman sera to IgG, IgA, IgM, complement, fibrinogen, and glomerular basement membrane. The specificity of each antiserum was tested by immunoelectrophoresis and Ouchterloney's gel precipitation technique.

\section{Clinical Features and Laboratory Findings}

All the seven patients presented as cases of the nephrotic syndrome. Their ages varied between 9 and 23 years (four patients were under 15 years of age). Three patients gave a history of frequent sore throat before the onset of the symptoms, but no laboratory evidence was found to suggest a streptococcal infection. Oedema was the presenting symptom in all the seven patients. Four patients (children) had moderate to severe oedema of both face and legs. In the remaining three patients (adults) it was of lesser severity and was confined to either the periorbital region or ankles. Two (children) of the seven patients showed evidence of microscopical haematuria. No evidence of hypertension was found in any of the patients. The duration

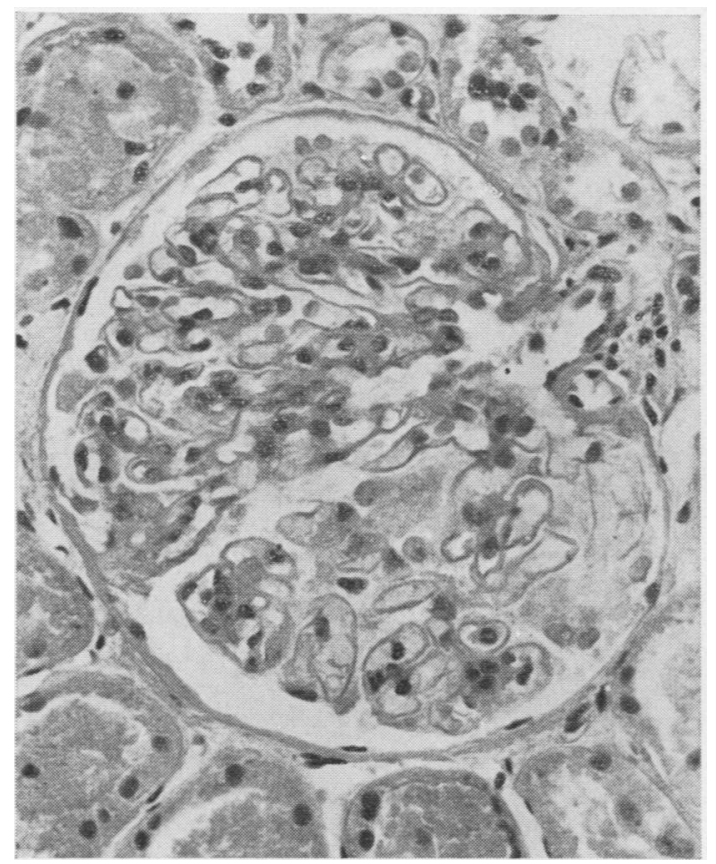

Fig. 1 Glomerulus. Focal sclerosis and capsular adhesion involving two to three loops. Haematoxylin and eosin. $\times 410$. of illness at the time of biopsy varied between two months and 14 months.

The 24-hour urinary protein excretion was higher in children than in adults. It varied between 4.1 and $15.7 \mathrm{~g}$ per 24 hours. Total serum proteins were less than $3.9 \mathrm{~g} / 100 \mathrm{ml}$ of serum in five out of seven patients. Serum cholesterol levels were above 285 $\mathrm{mg} \%$ in six of the seven cases. The glomerular filtration rate was assessed by creatinine clearances which were found to be less than $60 \mathrm{ml} /$ minute in the majority of cases. Blood urea levels varied between 52 and $82 \mathrm{mg} \%$.

\section{Results}

\section{HISTOLOGY}

The severity of histological changes varied with the duration of illness. The early lesion (one case) consisted of focal thickening of the capillary wall in the free loops of a few glomeruli. In two cases glomeruli showed adhesions to the Bowman's capsule. The lumens of the capillaries involved were partially or completely obliterated (Fig. 1). In three cases the sclerosis involved one or two lobules of up to $40 \%$ of the glomeruli (Fig. 2). In one case all the above mentioned changes were seen and a few glomeruli were completely sclerosed (Fig. 3).

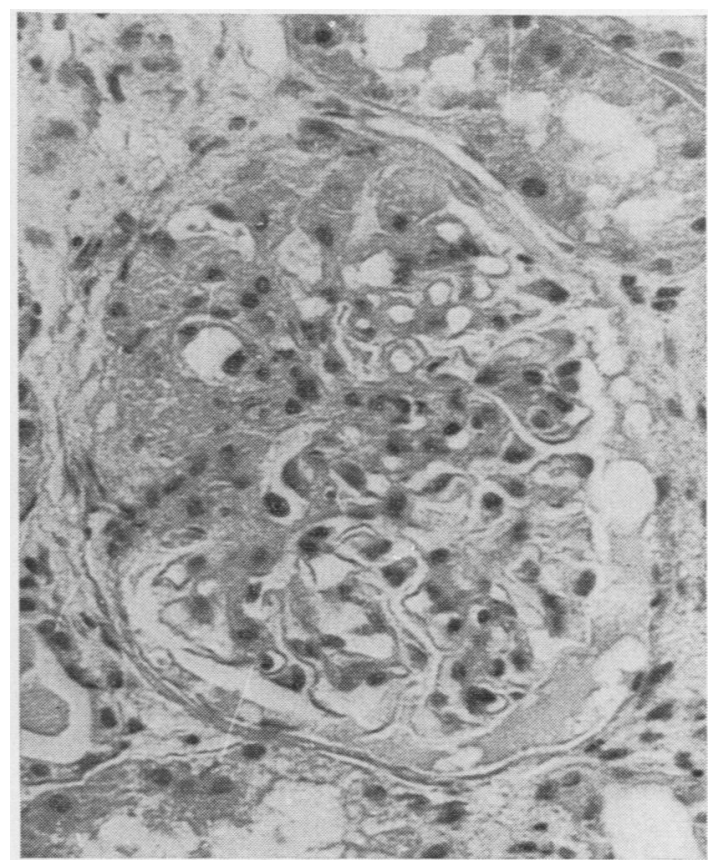

Fig 2 Glomerulus. Capsular adhesions and focal glomerular sclerosis involving three lobules. Haematoxylin and eosin. $\times 410$. 


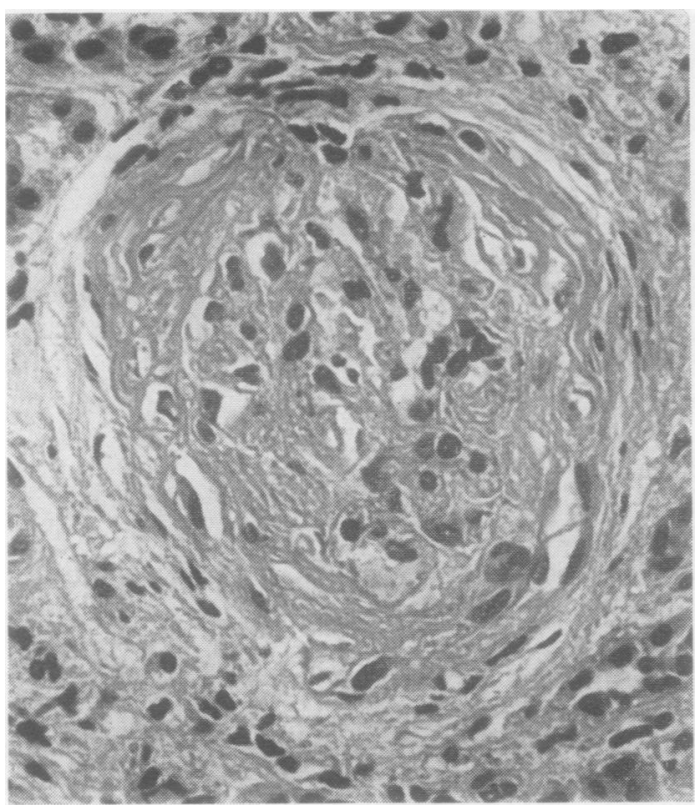

Fig. 3.

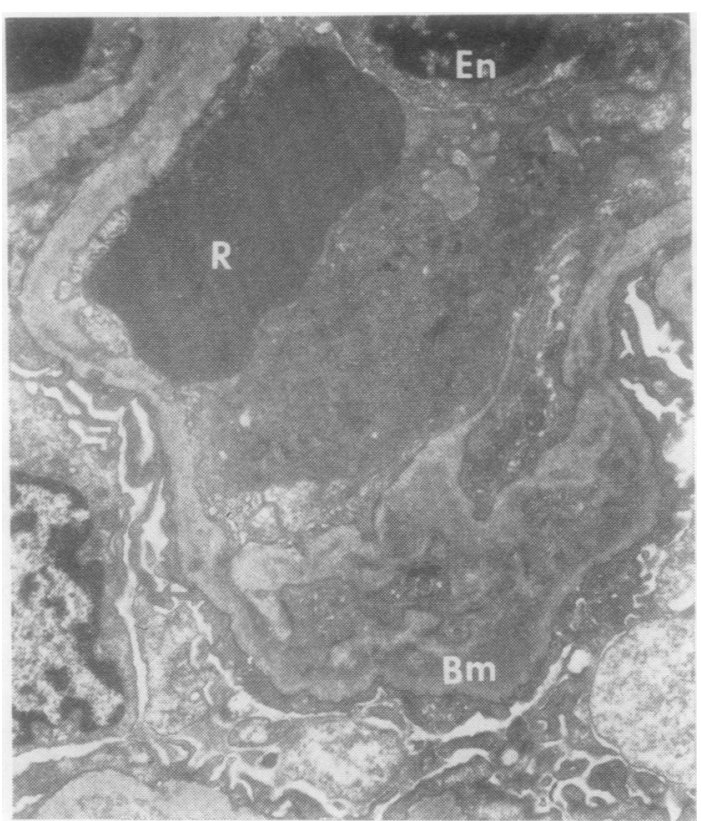

Fig. 4.

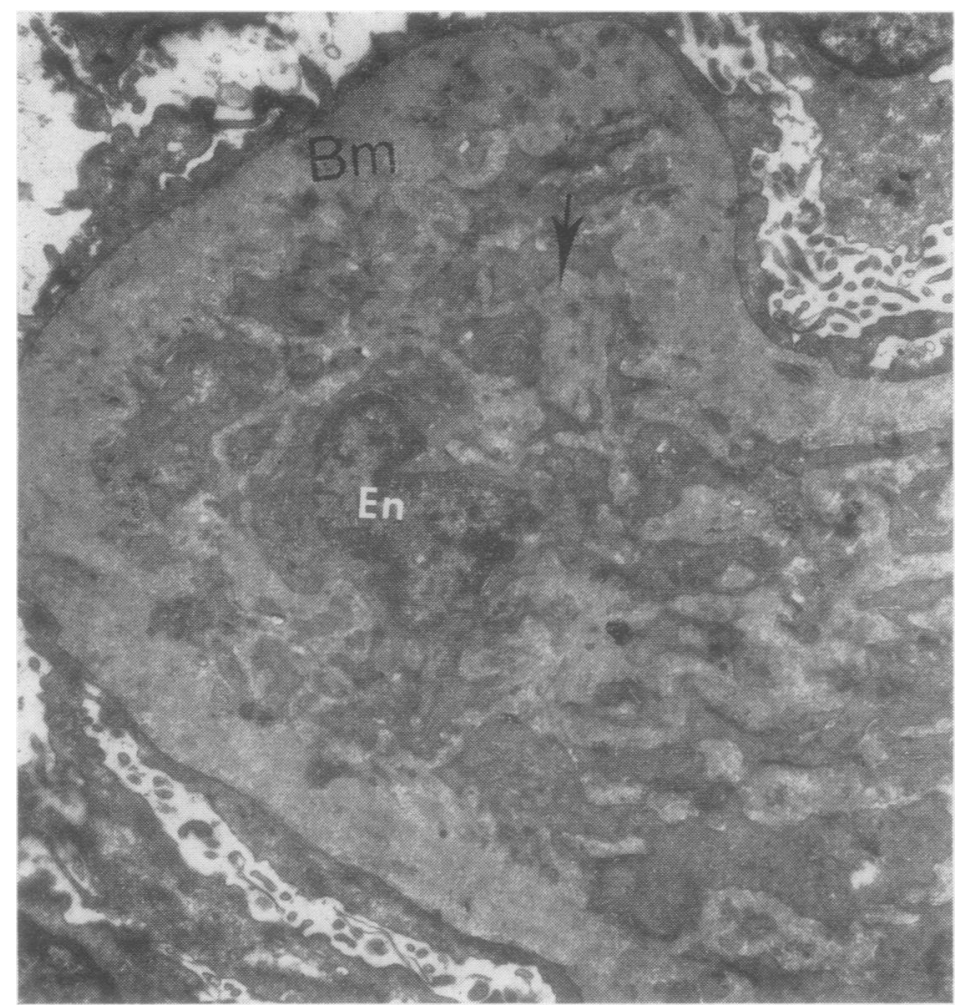

Fig. 3 Glomerulus. Sclerosis has involved the whole glomerular tuft with complete obliteration of capillary lumens. Haematoxylin and eosin. 460.

Fig. 4 A glomerular capillary loop containing a red blood cell $(\mathrm{R})$ and an endothelial cell (En). The

basement membrane $(\mathrm{Bm})$ in a portion of it is thickened towards the endothelial aspect. 5,600 .

Fig. 5 A glomerular capillary loop showing grossly thickened basement membrane $(\mathrm{Bm})$. The capillary lumen containing an endothelial cell (En) is completely obliterated by basement membrane-like material (arrow). 6,100 .

Fig. 5. 


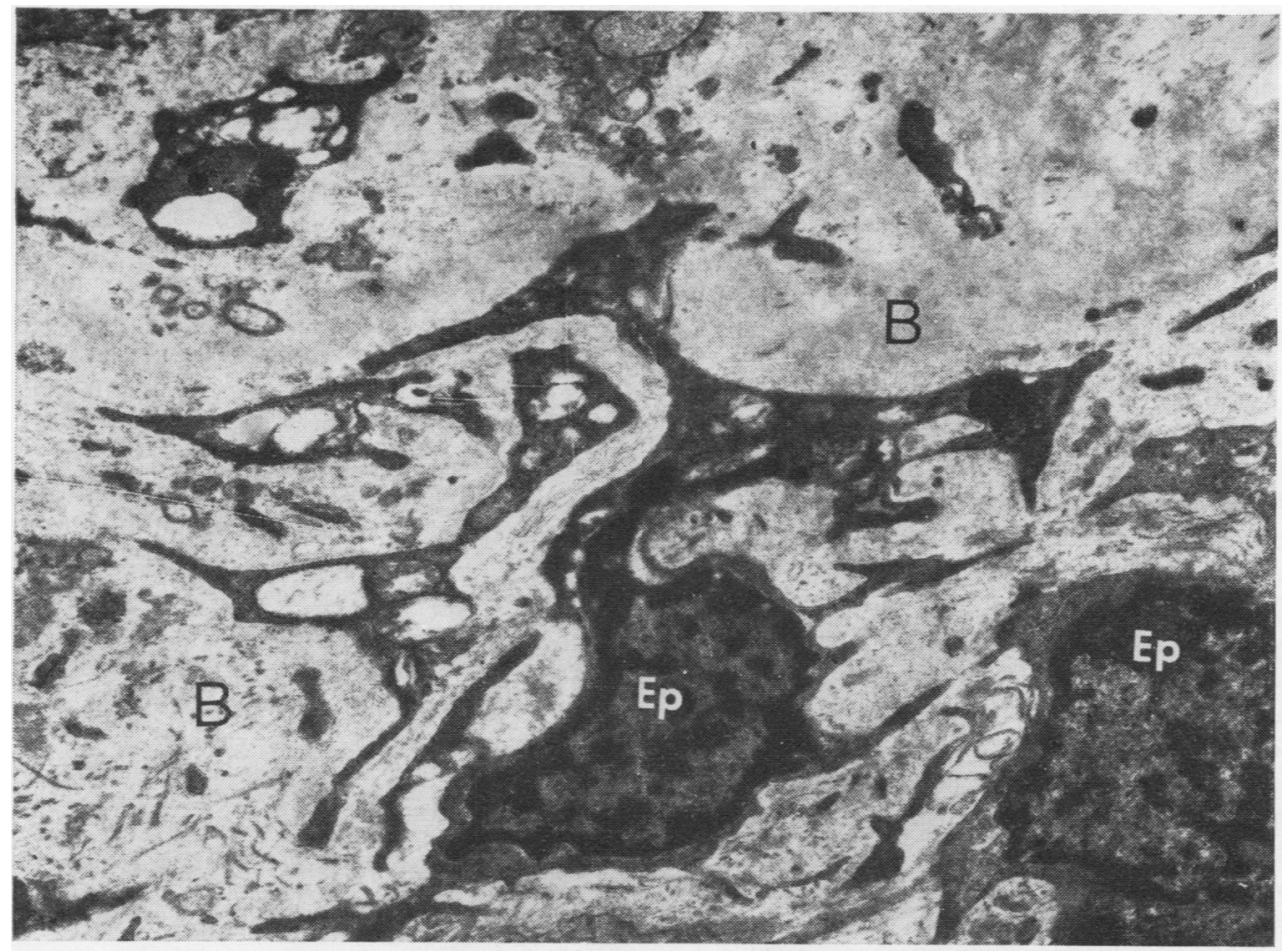

Fig. 6 Portion of a completely sclerosed glomerulus, showing large masses of basement membrane-like material (B). Two epithelial cell $(\mathrm{Ep})$ nuclei are seen compressed by the surrounding tissue. $\times 6,900$.

Methenamine-silver stained sections revealed that the sclerosed tissue mainly consisted of basement membrane-like material. The tubular atrophy and the interstitial fibrosis were seen only in relatively advanced cases.

All the unaffected glomeruli showed the features of a minimal change lesion.

\section{ELECTRON MICROSCOPY}

Electron microscopy was done in all the seven cases. Glomerular basement membrane in the mildly affected case showed slight to moderate irregular thickening in most of the loops (Fig. 4). However, in some of the loops the basement membrane was slightly darker in appearance, but did not show any definite deposits. In more advanced cases the basement membrane was much thickened and the capillary lumens were completely obliterated by basement membrane-like material (Fig. 5). The completely sclerosed glomeruli were composed of basement membrane-like material and some collagen fibres (Fig. 6). All the capillaries were compietely obliterated in the affected glomeruli. None of them showed electron-dense deposits in relation to the glomerular basement membrane.
The affected tubules showed diffuse thickening of the basement membrane. Their epithelial cells contained numerous protein reabsorption droplets.

\section{FLUORESCENT MICROSCOPY}

Immunofluorescent microscopy was carried out on all the seven cases. None of them showed the presence of gamma globulins or fibrin in the glomeruli.

\section{RESPONSE TO TREATMENT}

All seven patients were treated with corticosteroids. One patient with an early lesion showed an initial response to treatment, but soon became resistant to steroids. The remaining six patients showed a very poor or no response to the treatment.

\section{Discussion}

Focal glomerular sclerosis has recently been shown a good deal of attention due to its distinct morphological appearances and its poor response to steroid therapy. Rich (1957), who originally described this lesion, thought that in some lesions of minimal change, the glomeruli in the juxtamedullary region show focal thickening of the basement membrane. 
With the passage of time glomerular basement membrane became increasingly thickened with focal hyaline deposits in the tufts, leading gradually to complete obliteration of the normal glomerular architecture. Later on, Williams (1965), McGovern (1967), White (1970), White et al (1970), and Churg et al (1970) also studied this lesion and agreed with Rich's view that in the early stages this lesion may be difficult to distinguish from pure minimal change. We encountered similar difficulty in one of our cases, biopsied two months after the onset of symptoms. Histologically there was focal thickening of the capillary wall in the free loops of a few glomeruli. Electron microscopy revealed a definite thickening of the glomerular basement membrane in many loops. The remaining patients, who were biopsied between five months to 14 months after the onset of symptoms, could be diagnosed by histological examination alone.

Electron microscopical studies of renal biopsies of various stages of focal glomerular sclerosis may help in a better understanding of the disease process and in the correct diagnosis of early cases. The results of electron microscopical examination in our cases show that the severity of the glomerular lesion depends mainly on the severity of changes in the basement membrane. Regarding the nature of glomerular scar tissue, our electron microscopical observations suggest that the scar (sclerosed) tissue is formed mainly by basement membrane material which very gradually increases in amount to obliterate the capillary lumens. The other important component is mesangial matrix which also increases in amount and helps in obliterating the capillary lumens. The collagen fibres, although seen occasionally in some cases, do not play an important part in the formation of glomerular sclerosis. A similar $\stackrel{\text { 을 }}{\text {. }}$ view was held by Jones (1963) who studied the nature of scar tissue in glomerular diseases.

The most important reason for diagnosing and $\bar{C}$ identifying an early lesion of focal glomerular 흠 sclerosis is to obtain information regarding treatment $\stackrel{\frac{D}{D}}{\vec{D}}$ in these patients. It has already been reported $\stackrel{\mathbb{Q}}{\Omega}$ (Churg et al, 1970; White et al, 1970) that cases of $ळ$ focal glomerular sclerosis are resistant to steroid $\vec{A}$ therapy. The findings in our cases were similar to those shown by the above workers.

On the basis of early glomerular basement membrane changes and the poor response to steroid therapy and in the presence of a short history in some of our patients, it is suggested that focal $\vec{c}$ glomerular sclerosis perhaps starts as an independent $\infty$ disease process rather than as a variation or a later $\vec{\circ}$ stage of the minimal change lesion. The possibility of an immune mechanism playing a part in its $\rightarrow$ pathogenesis is difficult to believe in the absence of any immune complexes or gamma globulins in the $\overparen{\mathbb{Q}}$ glomeruli of these cases.

\section{References}

Churg, J., Habib, R., and White, R. H. R. (1970), Pathology of the nephrotic syndrome in children. Lancet, 7, 1299-1302.

Jones, D. B. (1963). The nature of scar tissue in glomerulonephritis. Amer. J. Path., 42, 185-199.

McGovern, V. J. (1967). Glomerulonephritis. Path. Ann., 2, 1-30.

Rich, A. R. (1957). A hitherto undescribed vulnerability of the juxtamedullary glomeruli in lipoid nephrosis. Johns Hopk. Hosp. Bull., 100, 173-186.

White, R. H. R. (1970). Glomerulonephritis in children. Brit. J. hosp. Med., 3, 746-756.

White, R. H. R., Glasgow, E. F., and Mills, R. J. (1970). Clinicopathological study of nephrotic syndrome in childhood. Lancet, 1, 1353-1359.

Williams, A. L. (1965). Renal biopsy in nephrotic syndrome in childhood. (Paper given at 10th Annual Meeting of the College of Pathologists of Australia). 